\title{
Criticism of Conventional Methods of Treating Solid Tumours in Man
}

\author{
G. C. CRILE, JUN., ${ }^{*}$ M.D.
}

The title of this paper was not my invention; it was assigned to me. It implies that I am not only critical of the conventional treatments of solid tumours, which in some respects I am, but also that I have devised better ways of treating them, a point about which I am not sure. Nothing short of extensive randomized studies will determine what kind of treatments are best from the standpoint of cure. Nevertheless, until it is proved that radical operations and prophylactic irradiation therapy are superior to simpler forms of treatment it is better to select the treatment that leaves the patient the least deformed and the most comfortable. In patients with cancer of the breast, for example, there is little or no difference between the rates of survival of patients treated by radical operations as compared to simple ones. Since many patients fear the deformities inflicted by radical mastectomy and irradiation almost as much as they do the disease, probably if nothing but simple treatments were advised patients would come to the surgeon earlier and the rate of cure might be increased.

\section{Laboratory Evidence Supporting Conservative Treatment}

The fact that cancer cells are distributed systemically early in the disease can be shown in inbred mice by implanting a metastasizing type of cancer on the foot. If the foot bearing the tumour is amputated before day 12 , metastases rarely appear and most of the mice are cured. But if at this time blood is taken from either the aorta or vena cava or if lung tissue taken from the tumour-bearing mice is injected into the muscles of the thigh of new mice of the same strain, the tumour is nearly always transferred. Hence, though cancer cells may be present in both blood and lungs, removal of the tumour may still prevent metastasis and effect a cure.

Cancer cells may lie dormant in the liver of rats for long periods of time yet never grow into visible tumours unless they are stimulated by one of a variety of physical or chemical means. ${ }^{1}$ As long as six months after the cells were introduced into the portal vein their growth in the liver could be stimulated by trauma to the liver. Similar dormancy of cancer cells has been demonstrated in the lungs of mice. ${ }^{2}$

If the cells shed from human cancers behave like those of cancers in mice clearly there is a critical period, after a primary tumour is treated, in which the cancer cells that are diffused widely throughout the body may or may not grow into metastases, depending on the resistance of the host. This resistance appears to be, in part at least, immunologic.

When the allogeneic tumour, S180, is implanted on the left hind foot of a Swiss mouse, the mouse soon becomes immune to reimplantation of the tumour. However, if the tumourbearing foot is amputated on day 10 , and four days later the contralateral foot is challenged by a dose of 800,000 tumour cells, $60 \%$ of the mice are susceptible to reimplantation. Hence immunity to reimplantation of a tumour disappears rapidly after the primary tumour is removed. Since in mice viable cells are still present in the lungs after the primary tumour has been removed, the fate of these cells is of crucial importance to survival.

On the other hand, if the foot bearing the tumour is treated by a single destructive dose of radiation the immunity to the S180 tumour persists for at least three weeks. The tumour cells are doomed to die as a result of the treatment, but they maintain their antigenicity and prolong the mouse's systemic immunity to the tumour during the period that the tumour is * Professor of Surgery, Cleveland Clinic, Cleveland, Ohio, U.S.A. disappearing. With another tumour system the incidence of metastasis is nearly doubled when the foot carrying the tumour is amputated as when it is irradiated. ${ }^{2}$ Hence does complete removal of a primary tumour at a time when cancer cells are present in the blood and are already lodged in the lungs stimulate the growth of metastases? Perhaps it would be preferable to treat the tumour by a single destructive dose of radiation, limited to the area of the primary tumour, and two or three weeks later to excise the irradiated tissue before it undergoes necrosis.

In 1954 Mitchison $^{3}$ showed that early in the growth of an allogeneic tumour immunity to the tumour could be transmitted to a new mouse of the same strain by transplanting to it several of the lymph nodes that drained the tumour. Neither contralateral nodes nor cells from blood or spleen transferred the immunity. Experiments along the same line led Billingham and his associates" to conclude that "the regional nodes are therefore held to be the principal but not the only seat of the reaction against skin homografts."

At the Cleveland Clinic we investigated the other side of this coin. In short we found that removing the regional nodes resulted in an incidence of distant metastases that was significantly higher than occurred when only the tumour was removed. ${ }^{5}$

\section{Clinical Evidence Supporting Conservative Treatment}

There is a widespread belief among laymen that operations for cancer promote the spread of the disease. Some doctors share this belief, for they have observed patients who have had cancers of the breast that appeared to be localized for many years and in whom metastases appeared soon after the cancer was removed. Many years ago Ferguson ${ }^{6}$ noted that the time of appearance of pulmonary metastases from osteogenic sarcomas of extremities correlated more closely with the time of amputation than with the time of the first appearance of the tumour, even when there was considerable delay in treatment.

Regardless of whether these impressions are valid, they should not be interpreted as an indication to leave cancers untreated. The longer a cancer grows the more apt it is to metastasize, even if these metastases do not appear until after the tumour is removed. Thus the best hope for cure lies in early treatment. The only question is whether the initial treatment should be an operation which removes the main source of the antigen that is necessary to maintain immunity against metastases, and which in some cases may be the means by which enhancing antibody is absorbed. When this circulating antibody coats the cells of the metastases it may conceal them from detection and destruction by immunocytes and thus result in enhancement of their growth.

If a radical operation removes the regional lymph nodes as well as all the primary tumour it may further impair the ability of the host to produce or maintain systemic immunity against the implantation and growth of metastases. Such immune reactions are present in many human cancers. Black and his associates ${ }^{7}$ found that when cancers of the breast showed lymphocytic infiltration of the primary tumour and the regional lymph nodes hyperplasia (sinus histiocytosis)both reactions indicating an immunologic response-the prognosis of the patients was much better than when these signs were lacking. Southam and Brunschwig, ${ }^{8}$ moreover, showed that most patients, even when suffering from advanced and incurable cancers, were immune to reimplantation of their 
own tumours. Old and Boyse ${ }^{9}$ have summarized the situation by saying, "It has become clear that some tumours may be regarded as homografts in the original hosts and that the progressive growth of a tumour does not necessarily indicate the absence of specific immunologic reactions."

Against this background it is interesting to summarize some of the results obtained at the Cleveland Clinic following simplified treatments which many surgeons would consider to be totally inadequate, yet which have yielded results that are equal to or superior than most of those reported following radical and ultraradical operations with or without irradiation.

\section{Simplified Treatment of Breast Cancer}

Sixteen years ago at the Cleveland Clinic a prospective study was undertaken on the treatment of breast cancer. I abandoned the conventional radical mastectomy and prophylactic irradiation, and my associates continued to employ it. The experiment lasted only three years before my associates also decided to abandon the conventional forms of treatment. During that period the rate of survival after simplified procedures-either simple mastectomy or local excision usually (unless nodes were palpably involved) without irradiation-were better at both five and ten years than after radical operations, after which a higher proportion of the patients were irradiated. ${ }^{10}$

Subsequently from 1957 up to 1964 only $1 \%$ of our patients with operable breast cancer were treated by the conventional radical mastectomy. Clearly the results are better than those usually reported following radical mastectomy with or without irradiation (Tables I and II).

TABLE I.-Treatment of Breast Cancer (1957-64 inclusive) in 401 Patients

\begin{tabular}{|c|c|c|}
\hline Treatment & No. of Patients & $\%$ of Patients \\
\hline $\begin{array}{l}\text { Radical mastectomy .. } \\
\text { Modified radical mastectomy } \\
\text { Simple mastectomy or local excision } \\
\text { Ccbalt (postoperative) }\end{array}$ & $\begin{array}{r}4 \\
179 \\
218 \\
84\end{array}$ & $\begin{array}{l}1 \\
45 \\
54 \\
21\end{array}$ \\
\hline
\end{tabular}

TABLE II.-Five-year Survival of Patients with Breast Cancer (1957-64)

\begin{tabular}{|c|c|c|c|}
\hline & $\begin{array}{l}\text { No. of } \\
\text { Patients } \\
\text { Alive }\end{array}$ & $\begin{array}{c}\text { Total } \\
\text { No. of } \\
\text { Patients }\end{array}$ & $\begin{array}{c}\% \\
\text { of } \\
\text { of } \\
\text { Patients }\end{array}$ \\
\hline 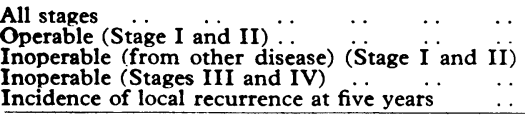 & $\begin{array}{r}300 \\
287 \\
0 \\
13 \\
25 \\
\end{array}$ & $\begin{array}{l}510^{*} \\
401 \\
8 \\
101 \\
401 \\
\end{array}$ & $\begin{array}{r}59 \\
72 \\
0 \\
13 \\
6\end{array}$ \\
\hline
\end{tabular}

- 507 of the $\mathbf{5 1 0}$ patients were followed for five or more years.

In Stage II it seemed to make little difference to the survival of the patients regardless of whether axillary nodes were removed or irradiated. But in true Stage I after radical operations when there was no involvement of nodes (as proved by the pathologist) or after simple operations when absence of involvement was proved by failure of the nodes to become palpable, the rate of survival was $15 \%$ higher when the nodes were not removed or irradiated. ${ }^{10}$

\section{Simplified Treatment of Papillary Thyroid Cancer}

Papillary cancer of the thyroid is unique among cancers, because it is the only one-with the exception of an occasional prostatic cancer-in which permanent regression can be induced by altering the hormonal environment. Hence in this disease reliance is placed more on the manipulation of hormones than on the preservation of immunity. In 18 of 31 patients seen at the Cleveland Clinic with pulmonary or other inoperable metastases of papillary carcinoma, the metastases have regressed or remained unchanged for from 5 to 27 years in response to feeding suppressive doses of thyroid hormone. ${ }^{11}$

Many surgeons view papillary carcinoma of the thyroid as a biological entity and treat almost all patients by total thyroidectomy and radical neck dissection. This might be justified in the treatment of some men over 40 who give no remote history of having had irradiation for a benign disease of the head or neck, for they are apt to have infiltrating metastasizing and autonomous cancers. Nevertheless, the girls and young women who constitute the majority of patients with papillary carcinoma-and who usually give a history of irradiation-have cancers that rarely metastasize outside the neck, tend to be non-invasive of surrounding tissues, and usually regress in response to oral doses of thyroid. Conservative operations followed by a lifetime programme of giving thyroid cures $99 \%$ of this group. The hospital mortality following radical operations on these patients would be higher than the death rate from cancer after conservative treatment. In a personal series of 231 patients seen between 1937 and 1964 only $2 \%$ have died of cancer. Between 1960 and 196490 patients were treated; of the 87 followed for from five to ten years none has died of cancer and only one is living with progressing disease. Only two total thyroidectomies and one block dissection of the neck were done. Seventy-one per cent. of the patients had metastases in the cervical nodes, but only $9 \%$ of the patients had to have a second operation for metastases in nodes outside the original field. These results were obtained despite the fact that almost all the operations were done through thyroidectomy incisions and that muscles and nerves were spared. Two patients when first seen had tumours so extensive that they were judged to be inoperable, and no attempt was made to remove them. Three more had preoperative pulmonary metastases. The tumours of all five of these inoperable patients have shrunk in resnonse to oral thyroid and the patients are well. One boy, aged 11, who had what was considered by another surgeon who removed most of the tumour to be an inoperable cancer infiltrating the trachea, was advised by a distinguished professor of surgery to have a total laryngectomy and bilateral neck dissection. Instead he was given 3 grains of thyroid daily and is well 10 years later without palpable recurrence.

\section{Conclusions}

If there is any criticism that I could make of the conventional methods of treating solid tumours, it would be that surgeons tend to think in terms of surgical techniques and that they often disregard the physiological and immunological principles that are so important in the control of the growth of a cancer. In some types of cancer (such as squamous cell cancer of the head and neck), from which distant metastases are uncommon, and four which there is no known method of hormonal control, radical surgery is as effective as anything known. Even in squamous cell cancer, however, probably preoperative radiation or a staging of the attack on the tumour and its secondary deposits might improve the results. In short, cancer can no longer be viewed as a devil to be exorcised as quickly and as radically as possible. To obtain the best results in patients with cancers in all of their various forms and stages, the attack should be varied to suit the needs of the individual. The day of speaking of the principles of cancer surgery is past. We must consider each cancer to be a biological entity in itself.

\section{REFERENCES}

1 Fisher, B., and Fisher, E. R., Annals of Surgery, 1955, 150, 731

2 Crile, G., jun., and Deodhar, S. D., Cancer. To be published.

3 Mitchison, N. A., Proceedings of the Royal Society Series B, 1954, 142 72.

Billingham, R. E., Brent, L., and Medawar, P. B., Proceedings of the Royal Society, Series B 1954, 143, 58

Crile, G., jun., Surgery, Gvnecology and Obstetrics, 1965, 120, 975

6 Ferguson, A. B., Fournal of Bone and Foint Surgery, 1940, 22, 92.

7 Black, M. M., Opler, S. R., and Speer, F. D., Surgery, Gynecology, and Obstetrics, 1955, 100, 543 .

8 Southam, C. M. and Brunschwig, A., Cancer, 1961, 14, 971.

- Old, L. J. and Boyse, E. A., Annual Review of Medicine, 1964, 15, 167

10 Crile, G iun Annals of Surgerv, 1968, 168, 330

11 Crile 722 . 